\title{
The Use of Color in Elemental Maps and Electron-Microscope Images.
}

\author{
R.F. Egerton \\ 1. Physics Department, University of Alberta, Edmonton, Canada T6G 2E1.
}

Color is used in elemental maps to provide a convenient way of displaying the results of electron-beam microanalysis. But such maps lack the intuitive appeal of gray-scale images in one important respect: there is no established consensus regarding the color assigned to each element. What are the prospects for standardization and how much information (including elemental concentration?) can be represented?

Chemists have adopted a few color choices in connection with molecular modeling, such as the CPK scheme [1,2]; see Table 1. Some of these choices are mnemonic, for example white for colorless hydrogen, black for carbon, yellow for sulfur, green for chlorine. However, the CPK scheme is not universal and it covers only a small fraction of the common elements, which raises the question of how many elements could be represented if a standard color scheme were to be agreed upon.

It has been claimed that the human eye can distinguish thousands or even millions of colors. Indeed, the standard 8-bit RGB scale allows $\left(2^{8}\right)^{3}=16,277,216$ colors to be represented in a computer-stored image. However, the color differences involved (when the R, G or B value is changed by 1 bit) are subtle and often undiscernable. Ignoring $5 \%$ of the population (mainly males) who are color-blind, humans have trichromatic color vision. This three-input limit greatly restricts the number of distinguishable colors, compared to 1024-channel spectral analysis for example; there are many different spectra (metamers) that give rise to the same color sensation.

Moreover, the number of colors that can be recognized (and assigned to a single element) is nodoubt a small fraction of the number than can be distinguished in a side-by-side comparison. In fact, it seems unlikely that all of the 92 elements would be individually recognizable. Although it is always possible to provide a key alongside the elemental map, there seems to be some consensus in the MSA community that the adoption of a few standard choices (as in Table 1) would be a step forward.

A related problem is what to do when different elements overlap. Without precautionary measures, equal amounts of oxygen (red) and halogen (green) would create (through additive mixing on a display screen) yellow, which might be mistaken for sulfur. One solution (at the expense of spatial resolution) is to use a patterned texture whose cells are based on the elements present in each region.

A different use of color is to represent the local intensity in an electron-microscope (TEM, SEM) image. Because the human eye can barely distinguish 16 grey levels [3], color allows more information to be represented in a single display. Although the choice of a false-color scheme is in principle arbitary, some choices are more logical, more intuitive, or more pleasing than others. One logical scheme represents increasing intensity by near-spectral colors from red to blue (as in Fig. 1a), corresponding to increasing photon energy or increasing temperature of an emitting object. However, many people associate red with heat, so an inverse-spectral scheme (blue to red; Fig. 1b) is probably more intuitive. The so-called hypsometric/bathymetric scheme is often used in cartography (deep blue for ocean depths, green for low-lying land, brown for higher, purple and white for mountains) and can be both pleasing and intuitive (Fig. 1c). 
Some of these color schemes allow a larger number of distinguishable levels than others. The eye appears to be more sensitive to small variations in the case of pale colors (center of the color triangle) and in the green region; see Fig. 2. In fact, pure spectral colors need to be avoided because they cannot be displayed via printing or on a RGB display such as a computer monitor [4].

References:

[1] L Corey and L. Pauling, Rev. Sci. Instrum. 24 (1953), p. 621.

[2] WL Koltun (1965), U. S. Patent 3170246.

[3] AV Crewe, Scanning 3 (1980), p. 176.

[4] The author thanks the Natural Sciences and Engineering Research Council of Canada for funding.

\begin{tabular}{|c|c|c|c|}
\hline element & atomic no. & Corey and Pauling (1953) & Koltun (1965) \\
\hline hydrogen & 1 & white & white \\
\hline carbon & 6 & black & black \\
\hline nitrogen & 7 & blue & red \\
\hline oxygen & 8 & red & purple \\
\hline phosphorus & 15 & -- & yellow \\
\hline sulfur & 16 & -- & shades of green \\
\hline halogens & $9,17,35,53$ & -- & silver \\
\hline metals & $26-29$ & -- & . \\
\hline
\end{tabular}

Table 1. CPK color assignments, based on Corey and Pauling [1] and on Koltun [2].

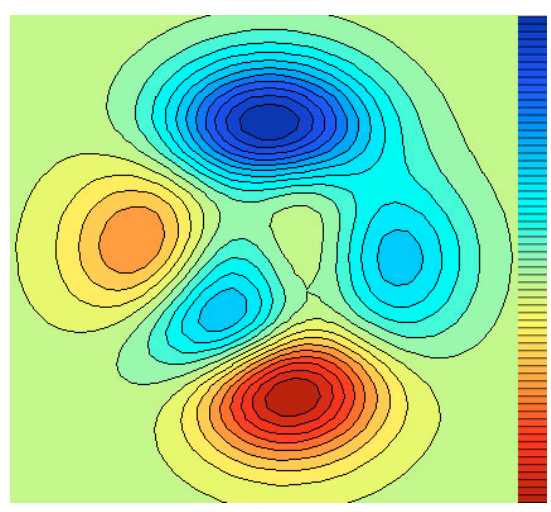

(a)

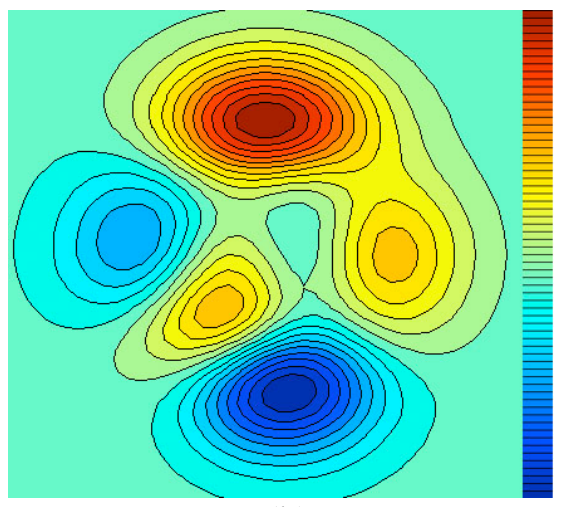

(b)

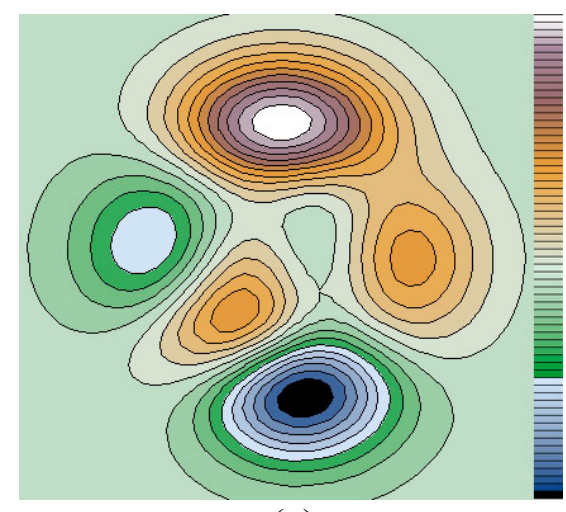

(c)

Figure 1. Contour image showing three peaks and two valleys, in three color schemes: (a) with red to blue representing increasing height, (b) with blue to red representing increasing height, (c) with a hypsometric/bathymetric scheme, as used in cartography. The color key is to the right of each image.

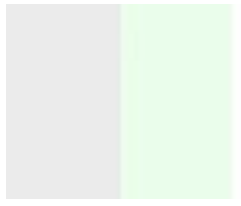

(a)

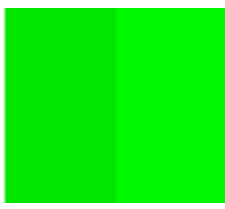

(b)

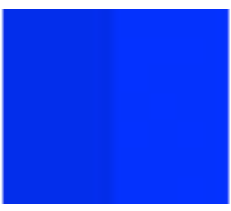

(c)

Figure 2. Effect of (a) increasing the green content of pale gray by $10 \%$, (b) increasing the intensity of green by $10 \%$, and (c) increasing the intensity of blue by $10 \%$. 